\title{
Degradation of a quantum directional reference frame as a random walk
}

\author{
Stephen D. Bartlett, ${ }^{1}$ Terry Rudolph, ${ }^{2,3}$ Barry C. Sanders, ${ }^{4,5}$ and Peter S. Turner ${ }^{4}$ \\ ${ }^{1}$ School of Physics, The University of Sydney, Sydney, New South Wales 2006, Australia \\ ${ }^{2}$ Optics Section, Blackett Laboratory, Imperial College London, London SW7 2BZ, United Kingdom \\ ${ }^{3}$ Institute for Mathematical Sciences, Imperial College London, London SWr 2BW, United Kingdom \\ ${ }^{4}$ Institute for Quantum Information Science, University of Calgary, Alberta T2N 1N4, Canada \\ ${ }^{5}$ Centre of Excellence for Quantum Computer Technology, \\ Macquarie University, Sydney, New South Wales 2109, Australia
}

(Dated: 31 January 2007)

\begin{abstract}
We investigate whether the degradation of a quantum directional reference frame through repeated use can be modelled as a classical direction undergoing a random walk on a sphere. We demonstrate that the behaviour of the fidelity for a degrading quantum directional reference frame, defined as the average probability of correctly determining the orientation of a test system, can be fit precisely using such a model. Physically, the mechanism for the random walk is the uncontrollable back-action on the reference frame due to its use in a measurement of the direction of another system. However, we find that the magnitude of the step size of this random walk is not given by our classical model and must be determined from the full quantum description.
\end{abstract}

\section{INTRODUCTION}

Reference frames are physical objects subject to the laws of physics like any other. Although it is common, especially within quantum physics, to treat reference frames as non-dynamical objects outside of the theory, there are a wide variety of situations where this assumption is not valid. Reference frames are quantifiable resources [1]: they are non-trivial to prepare and to share [2], their imperfections can result in deleterious consequences for performing high-precision measurements [3, 4, 5], and they can degrade with repeated use [6, 7].

The issue of degradation of a reference frame is particularly important for quantum computation, where a large number of high-precision measurements must be performed. In many implementations, such measurements are performed relative to a reference frame that is described by a finite quantum system; for example, a small-amplitude coherent state of the electromagnetic field [3, 4], the proposed single-spin measurement technique using magnetic resonance force microscopy [8], or the single-electron transistors used for measurement of superconducting qubits [9]. When measuring an ensemble of systems for which the mean polarization is precisely zero, the number of measurements for which a quantum reference frame for direction or for phase can be used has been shown to scale quadratically rather than linearly in the size of the reference frame [6]. This result provides promise for the use of microscopic or mesoscopic reference frames in performing repeated high-precision measurements where degradation of the reference frame is significant, provided that quantum information is encoded in states with zero mean polarization.

In [7], it was argued that this quadratic scaling behaviour is the result of fluctuations in the polarization of the measured systems. A primary motivation for the current paper was to address the question of whether the quadratic scaling behaviour was a manifestation of the well known quadratic behaviour of a classical random walk's mean displacement. In this paper we answer this question in the negative. Although a classical random walk does capture the functional form of the degradation process, the step size of the walk (which is what ultimately determines the quadratic scaling of the longevity described above) must be determined from the full quantum description. Once the step size is fit to the quantum model, however, the classical random walk determines at least one measure of the frame's degradation perfectly.

The degradation of a quantum directional reference frame (DRF) can be quantified by the decrease in measurement fidelity (the probability of correctly measuring a spin that is known to be aligned/anti-aligned with the frame) as a function of the number of previous measurements for which it has been used. Although the mathematical framework for calculating the decrease in measurement fidelity has been established [6], the simple connection with a random walk shown here provides two important advantages. One advantage is that the random walk is easier to model so the incorporation of reference frame degradation into quantum computing simulations will be simplified by this random walk analogy rather than having to account for a large Hilbert space. The second advantage is that the random walk analogy provides a simple and elegant conceptual framework for the the effects on reference frames by sequential measurements, which will make considerations of general problems involving reference frame degradation easier to comprehend and solve.

The structure of the paper is as follows. In Sec. II we review the results of the degradation of a quantum DRF [6]. In Sec. III] we present a semi-classical model for a DRF. We demonstrate how a classical model with some uncertainty in its direction can be characterized by a probability distribution on a sphere. We initiate our semi-classical DRF in a probability distribution that is comparable to the quantum DRF in [6]. At this point, in order to introduce a degradation due to its use in mea- 
surement, we construct a semi-classical measurement theory for the DRF based on two simple assumptions. We argue that this measurement theory leads to a spherical random walk on the probability distribution of this classical DRF. Finally, we show that the form of the degradation exactly matches that of quantum case found in [6], and that a single fitted parameter - the classical step size - yields a value of the classical degradation that exactly matches the quantum. Conclusions are presented in Sec. IV.

\section{DEGRADATION OF A QUANTUM DRF}

In this section, we review the results of [6], which characterize the degradation of a quantum DRF. The key result we will reproduce with a semi-classical model is the rate of decrease of the measurement fidelity in terms of the number of previous measurements for which the reference frame was used.

We use a spin- $j$ system for our quantum DRF, with Hilbert space $\mathbb{H}_{j}$. We choose the initial quantum state of the spin- $j$ system to be $\rho^{(0)}=|j, j\rangle\langle j, j|$, which was determined in [6] to be the initial state that maximizes the initial success probability. (This quantum reference frame is aligned in the $+z$ direction relative to a background DRF, which is essentially a choice of gauge.)

The systems to be measured against the quantum DRF will be spin- $1 / 2$ systems, each with a Hilbert space $\mathbb{H}_{1 / 2}$. We consider the situation wherein the initial state of each such system is the completely mixed state $I / 2$, where $I$ is the identity operator on $\mathbb{H}_{1 / 2}$. The use of maximally mixed qubits corresponds to having no information about the state of the spin- $1 / 2$ systems prior to measurement. In particular, this condition ensures that the qubits have zero mean polarization with respect to the quantum DRF, i.e., the qubits and the quantum DRF are initially completely uncorrelated. (Note that, if the measured systems have a non-zero mean polarization with respect to the DRF, even a very small one, the resulting measurements will induce a drift on the DRF resulting in a linear scaling [7].)

Our quantum DRF will be used to measure many such independent spin- $1 / 2$ systems sequentially. We shall assume trivial dynamics between measurements, and thus our time index will simply be an integer specifying the number of measurements that have taken place. The state of the DRF following the $n^{\text {th }}$ measurement is denoted $\rho^{(n)}$, with $\rho^{(0)}$ denoting the initial state of the DRF prior to any measurement.

A measurement of the relative orientation of a spin- $1 / 2$ particle to a spin- $j$ system is represented by operators that are invariant under collective rotations. The measurement that provides the maximum information gain about the relative orientation between a spin- $j$ and a spin-1/2 system has been determined in [10] to be a measurement of the magnitude of the total angular momentum $\hat{J}^{2}$. That is, it is the two-outcome projective mea- surement $\left\{\Pi_{+} \equiv \Pi_{j+1 / 2}, \Pi_{-} \equiv \Pi_{j-1 / 2}\right\}$ on $\mathbb{H}_{j} \otimes \mathbb{H}_{1 / 2}$.

The evolution of the DRF will depend on the measurement record. In general, even if one keeps the entire measurement record and determines the conditional state of the DRF based on this record, there will be a degradation of the DRF (specifically, a non-unitary evolution of the reduced density matrix describing the DRF) due to entanglement between the state of the DRF and the measured spins. The magnitude of this degradation will depend explicitly on the measurement record. In order to quantify the expected (or average) degradation, we consider averaging over all possible measurement records. Note that this averaging is equivalent to discarding the measurement record, and we consider this expected degradation in what follows. We note that an interesting avenue for future work would be to address the worst-case scenario as opposed to the average case.

The evolution of the quantum DRF as a result of the $n^{\text {th }}$ measurement is

$$
\rho^{(n+1)}=\mathcal{E}\left(\rho^{(n)}\right)
$$

where the superoperator $\mathcal{E}$ is given by

$$
\mathcal{E}(\rho)=\operatorname{Tr}_{S}\left(\sum_{c \in\{+,-\}} \Pi_{c}(\rho \otimes I / 2) \Pi_{c}\right),
$$

with $\operatorname{Tr}_{S}$ the partial trace over $\mathbb{H}_{1 / 2}$.

The map $\mathcal{E}$ can be written using the operator-sum representation [11] as

$$
\mathcal{E}(\rho)=\frac{1}{2} \sum_{\substack{c \in\{+,-\} \\ a, b \in\{0,1\}}} E_{a b}^{c} \rho E_{a b}^{c \dagger},
$$

where $E_{a b}^{c} \equiv\left\langle a\left|\Pi_{c}\right| b\right\rangle$ is a Kraus operator on $\mathbb{H}_{j}$ and $\{|0\rangle,|1\rangle\}$ is a basis for $\mathbb{H}_{1 / 2}$. These operators can be straightforwardly determined in terms of Clebsch-Gordon coefficients. The resulting evolution has not been solved analytically; a closed-form solution for the limiting case $j \gg 1$ is presented in Ref. [6].

We quantify the quality of a quantum DRF as the average probability of a successful estimation of the orientation of a fictional "test" spin- $1 / 2$ system which is, with equal probability, either aligned or anti-aligned with the background $+z$-axis. Denote the pure state of the test spin-1/2 system that is aligned (anti-aligned) with the initial DRF by $|0\rangle(|1\rangle)$. For a spin- $1 / 2$ system prepared in the state $|0\rangle$ or $|1\rangle$ with equal probability, the average probability of success using a quantum DRF state $\rho$ is

$$
F_{Q}=\frac{1}{2} \operatorname{Tr}_{R}\left(\rho\left(E_{00}^{+}+E_{11}^{-}\right)\right),
$$

where the subscript $Q$ denotes that this is a fully quantum result. We denote this quantity as the quantum average measurement fidelity.

Although Eq. (3) has not been solved analytically, it is still possible to obtain an exact expression for the evolution of the quantum average measurement fidelity under 
this evolution. In [6], it is found that the solution for $\rho^{(n)}$, given the initial state $\rho^{(0)}=|j, j\rangle\langle j, j|$, yields a quantum average measurement fidelity $F_{Q}(n)$ that decreases with $n$ as

$$
F_{Q}(n)=\frac{1}{2}+\frac{j}{2 j+1}\left(1-\frac{2}{(2 j+1)^{2}}\right)^{n}
$$

This expression, which we emphasize is exact, implies that the number of measurements for which an DRF is useful increases quadratically with $j$, the size of the reference frame.

\section{A SEMI-CLASSICAL MODEL}

In this section, we demonstrate that the complete quantitative behavior of the degradation of a DRF can be modeled as an ideal DRF undergoing a (classical) random walk on the sphere. For such a description, we will develop a semi-classical measurement theory - one that describes measurement with respect to an ideal reference frame about which there is some classical uncertainty, and which includes a back-action due to the measurement that randomly "kicks" the reference frame, yielding a random walk.

\section{A. Probability distributions of a DRF}

Consider an ideal classical DRF. (This DRF could be realized, say, by a spin system as described above with $j \rightarrow \infty$.) The possible configurations of this DRF are described by the unit sphere, $S^{2}$, with coordinates $(\theta, \phi)$ given by the polar angle $\theta \in[0, \pi]$ and the azimuthal angle $\phi \in[0,2 \pi)$. Such an ideal classical DRF allows for projective measurements to be performed in the angular momentum basis defined by $(\theta, \phi)$.

To model degradation of an ideal classical DRF, we consider classical probability distributions $p(\theta, \phi)$ on the unit sphere $S^{2}$. These probability distributions describe the experimenter's knowledge about the orientation of the classical DRF. The usual properties of probability distributions must apply, namely normalisation and positivity:

$$
\begin{gathered}
\int_{0}^{2 \pi} \frac{\mathrm{d} \phi}{2 \pi} \int_{0}^{\pi} \frac{\sin \theta \mathrm{d} \theta}{2} p(\theta, \phi)=1, \\
p(\theta, \phi) \geq 0 \quad \forall(\theta, \phi) \in S^{2} .
\end{gathered}
$$

All functions on the sphere can be expanded in terms of spherical harmonics $Y_{\ell m}(\theta, \phi)$. In this paper, we will only make use of distributions that are azimuthally symmetric, i.e., distributions that do not depend upon the azimuthal angle $\phi$. Such distributions can be expanded in terms of the spherical harmonics with $m=0$. Because $Y_{\ell 0}$ is proportional to the Legendre polynomial $P_{\ell}(\cos \theta)$, we have

$$
p(\theta)=\sum_{\ell=0}^{\infty} c_{\ell} P_{\ell}(\cos \theta) .
$$

Two Legendre polynomials that will play a major rôle in what follows are $P_{0}(x)=1$ and $P_{1}(x)=x$. The inner product

$$
\int_{0}^{\pi} \frac{\sin \theta \mathrm{d} \theta}{2} P_{\ell}(\cos \theta) P_{\ell^{\prime}}(\cos \theta)=\frac{1}{2 \ell+1} \delta_{\ell \ell^{\prime}},
$$

will also be useful.

\section{B. An initial distribution comparable with a finite quantum DRF}

We will initiate our classical DRF (at $n=0$, prior to the first measurement) aligned in the $+z$ direction, just as in the fully quantum description. (Again, this is essentially a choice of gauge.) Also, we choose an initial probability distribution that has classical uncertainties comparable with those of the initial quantum state $\rho^{(0)}=|j, j\rangle\langle j, j|$ that was used in Sec. [II. Noting that $\rho^{(0)}$ is invariant under rotations about the $z$-axis, the initial classical distribution should also be so, i.e., independent of $\phi$. To this end, we choose the initial classical distribution to be

$$
p^{(0)}(\theta)=(4 j+1)[\cos (\theta / 2)]^{8 j} .
$$

Equation (10) is a valid probability distribution, satisfying Eqs. (6) and (7), and which we now argue has the correct angular uncertainties, comparable with those of the quantum state $|j, j\rangle$.

The state $|j, j\rangle$ can be viewed as an $\mathrm{SU}(2)$ coherent state [12, 13] and saturates the angular-momentum uncertainty relations $\left(\Delta J_{x}\right)^{2}\left(\Delta J_{y}\right)^{2} \geq \frac{1}{4}\left\langle J_{z}\right\rangle^{2}$, with $\left(\Delta J_{x}\right)^{2}=\left(\Delta J_{y}\right)^{2}=j / 2$. The angular uncertainty (variance) of the state $|j, j\rangle$, then, is $(\Delta \theta)^{2}=\left(\Delta J_{x}\right)^{2} /\left\langle J_{z}\right\rangle^{2}=$ $1 /(2 j)$.

The distribution $p(\theta)$ possesses the same angular variance, as follows. For small $\theta$ and large $j$, we can use the approximation [14]

$$
[\cos (\theta / 2)]^{8 j} \rightarrow \exp \left(-j \theta^{2}\right) .
$$

Viewed as a Gaussian distribution for small $\theta$, this has variance $\sigma^{2}=1 /(2 j)$. Thus, the probability distribution of Eq. (10) is (i) aligned along the $+z$-direction, (ii) azimuthally symmetric, and (iii) has angular uncertainties that are equal to those of the quantum state $|j, j\rangle$.

\section{Semi-classical measurement theory}

We now introduce a semi-classical measurement theory that allows us to calculate the probability of correctly 
measuring a known spin, either aligned or anti-aligned with the background $z$-axis, using an ideal classical DRF with an uncertainty in its direction (i.e., described by a probability distribution).

\section{Measurement fidelity}

The measurement of a single spin-1/2 particle relative to an ideal classical DRF aligned along the $+z$ axis can be described as a projective measurement of the qubit in the $\{|0\rangle,|1\rangle\}$ basis. If this ideal classical DRF is misaligned by a known angle $\theta$ relative to the background $+z$-axis, the measurement of a spin$1 / 2$ particle relative to this misaligned DRF is also described by a projective measurement but now in the basis $\{(\cos (\theta / 2)|0\rangle+\sin (\theta / 2)|1\rangle),(\cos (\theta / 2)|1\rangle-\sin (\theta / 2)|0\rangle)\}$. Consider a spin- $1 / 2$ particle that is known to be aligned with the background $+z$-axis. The probability that a measurement of this particle relative to the misaligned frame will yield the correct result is $P=(1+\cos \theta) / 2=$ $\cos ^{2} \theta / 2$. Thus, a misalignment of an ideal classical DRF leads to a finite probability of error when measuring the direction of objects that are known to be aligned (or antialigned) to the $z$-axis.

Now consider extending this measurement theory to an ideal classical DRF with a distribution of misalignments $p(\theta)$ compared to the background $z$-axis. The probability of correctly measuring the direction of a spin- $1 / 2$ particle that is known to be aligned or anti-aligned to the $z$-axis is given by

$$
F_{C}=\int_{0}^{\pi} \frac{\sin \theta \mathrm{d} \theta}{2} p(\theta) \cos ^{2}(\theta / 2),
$$

which we will call the classical average measurement fidelity.

We can expand $p(\theta)$ in the basis of Legendre polynomials as in Eq. (8). Because $\cos ^{2}(\theta / 2)$ can be simply expressed in terms of Legendre polynomials as $\cos ^{2}(\theta / 2)=$ $\frac{1}{2}\left(P_{0}(\cos \theta)+P_{1}(\cos \theta)\right)$, and because the Legendre polynomials form an orthogonal basis, the classical average measurement fidelity depends only upon the $\ell=0$ and $\ell=1$ terms, i.e.,

$$
\begin{aligned}
F_{C} & =\int_{0}^{\pi} \frac{\sin \theta \mathrm{d} \theta}{2}\left(\sum_{\ell=0}^{\infty} c_{\ell} P_{\ell}(\cos \theta)\right) \cos ^{2}(\theta / 2) \\
& =\sum_{\ell=0}^{\infty} c_{\ell} \int_{0}^{\pi} \frac{\sin \theta \mathrm{d} \theta}{2} P_{\ell}(\cos \theta) \frac{1}{2}\left(P_{0}(\cos \theta)+P_{1}(\cos \theta)\right) \\
& =\frac{1}{2}\left(c_{0}+\frac{1}{3} c_{1}\right) .
\end{aligned}
$$

We note that this classical average measurement fidelity $F_{C}$ can be compared directly with the quantum average measurement fidelity $F_{Q}$ : both quantify the average probability of a successful estimation of a known spin that is either aligned or anti-aligned with the $+z$ axis.

\section{Back-action}

Our semi-classical measurement theory will also incorporate a form of back-action on the reference frame, i.e., an uncontrollable disturbance of the direction of the reference frame as the result of a measurement. A natural model for this back-action is as follows: as a result of a measurement of a spin, the state of the DRF is "kicked" in a random direction (sampled uniformly from the interval $[0,2 \pi))$ by a fixed angular distance $\alpha$. Repeated measurements using this DRF, then, can be modeled by a random walk on the sphere.

We note that the step size $\alpha$ is a free parameter in our measurement theory; it will be determined by fitting the behavior with the fully quantum evolution. However, it would be natural for this step size to vary as the ratio of the size of the system being measured to the size of the reference frame. In the quantum scenario that we wish to model, where $\hbar j$ is the angular momentum of the quantum reference frame and $\hbar / 2$ is the angular momentum of the spin- $1 / 2$ systems being measured, this ratio scales as $1 / j$. We will find that our fit to the quantum model agrees with this intuition.

\section{Classical random walk on the sphere}

We now consider the evolution of a probability distribution $p$ undergoing a random walk on the sphere [15]. Let the initial distribution be denoted $p^{(0)}$, and denote the distribution after $n$ steps by $p^{(n)}$. Our random walk on the sphere is defined such that, at each step, it is equally probable to make a step of fixed angular size $\alpha$ in any direction. The evolution of the probability distribution undergoing the random walk is then given by

$$
p^{(n+1)}(\theta, \phi)=\left[\hat{A}_{\alpha} p^{(n)}\right](\theta, \phi),
$$

where the operator $\hat{A}_{\alpha}$ averages its operand over all points that are one angular step $\alpha$ away from the target point $(\theta, \phi)$. For example, if the initial distribution is a delta-function $p_{\delta}(\theta, \phi)=\delta(\theta)$ at the north pole, after one step the distribution is the uniform average over the ring of points $\{(\alpha, \phi) \mid \phi \in[0,2 \pi)\}$.

Because the average of a sum is the sum of the averages, $\hat{A}_{\alpha}$ is linear and can be diagonalized. Assuming the step size $\alpha$ is fixed, the eigenfunctions are the spherical harmonics, with eigenvalues given by the Legendre polynomials [15],

$$
\left[\hat{A}_{\alpha} Y_{\ell m}\right](\theta, \phi)=P_{\ell}(\cos \alpha) Y_{\ell m}(\theta, \phi) .
$$

If we expand an initial azimuthally-symmetric distribution $p^{(0)}(\theta)$, expressed in the basis of Legendre polynomials as

$$
p^{(0)}(\theta)=\sum_{\ell=0}^{\infty} c_{\ell}^{(0)} P_{\ell}(\cos \theta)
$$


then the distribution after an $n$-step random walk is given by

$$
p^{(n)}(\theta)=\sum_{\ell=0}^{\infty} c_{\ell}^{(0)} P_{\ell}(\cos \theta)\left[P_{\ell}(\cos \alpha)\right]^{n} .
$$

The coefficients $c_{\ell}^{(n)}$ for the distribution at any timestep $n$ are therefore given in terms of the initial coefficients as

$$
c_{\ell}^{(n)}=c_{\ell}^{(0)}\left[P_{\ell}(\cos \alpha)\right]^{n} .
$$

This provides us with the classical evolution of the DRF.

\section{E. Fitting the quantum degradation to a random walk}

We now consider how the distribution $p^{(0)}(\theta)$ of Eq. (10), undergoing a random walk on the sphere with angular step size $\alpha$, serves as a DRF according to the classical average measurement fidelity as a figure of merit.

For the distribution $p^{(0)}(\theta)$ of Eq. (10) expressed as an expansion in terms of Legendre polynomials as in Eq. (8), the first two coefficients are found to be

$$
c_{0}^{(0)}=1, \quad c_{1}^{(0)}=\frac{6 j}{2 j+1} .
$$

The first two coefficients after $n$ steps of the random walk are given by Eq. (18) to be

$$
c_{0}^{(n)}=1, \quad c_{1}^{(n)}=\frac{6 j}{2 j+1} \cos ^{n} \alpha .
$$

The classical average measurement fidelity after $n$ steps, using Eq. (13), is thus

$$
F_{C}(n)=\frac{1}{2}+\frac{j}{2 j+1} \cos ^{n} \alpha .
$$

We note that this fidelity is precisely equal to that of the fully-quantum case, given by Eq. (5), if the step size $\alpha$ of the random walk is given by

$$
\cos \alpha=1-\frac{2}{(2 j+1)^{2}} .
$$

As argued above, the dependence on $j$ of this step size appears reasonable, satisfying $\alpha \simeq 1 / j$ for large $j$. However, we emphasize that this parameter is obtained from the full quantum theory, as it is the fundamental quantum back-action that determines its magnitude.

Given that the classical model reproduces the average measurement fidelity of the quantum model exactly, i.e., to all orders in $1 / j$ when a step size given by Eq. (22) is used, it is natural to question whether the full quantum evolution of the DRF is captured by our semi-classical model. Specifically, one might naively suggest that an initial SU(2) coherent state $|j, j\rangle$ undergoing a random walk on the sphere with step size (22) would yield a mixed quantum state of the DRF that provides a solution to the quantum evolution of Eq. (3). Surprisingly, this is not the case: we have checked numerically that solutions to Eq. (3) cannot be expressed as convex combinations of $\mathrm{SU}(2)$ coherent states. Thus, we emphasize that, while the degradation (as quantified by the average measurement fidelity) is captured precisely by our semi-classical model, the full evolution of the quantum DRF is not.

\section{CONCLUSIONS}

We have shown here that, given a suitable initial classical distribution for the misalignment of an ideal DRF and a reasonable semi-classical measurement theory that incorporates back-action, the exact expression of the decreasing measurement fidelity describing the degradation of a quantum DRF is reproduced by a classical random walk using a single fitted parameter - the step size of the random walk.

As the step size was fitted to the back-action from the full quantum result, a semi-classical argument on its own does not precisely explain the quadratic scaling of a reference frame's longevity [6]. However, one can provide an heuristic argument for quadratic scaling. Note that, at least in the case of a directional reference frame, it is the $1 / j$ behavior of the angle $\alpha$, that leads to quadratic scaling. The simple classical picture of adding a small vector randomly to a vector of length $j$ shows that the angle $\alpha$ between the resulting sum of vectors and the original vector will scale as $1 / j$, leading to a quadratic scaling in longevity.

We emphasize that our results are applicable only to the case where the measured systems have zero mean polarization relative to the DRF. A natural extension of this work would be to incorporate into this analysis the linear drift of the DRF that occurs when this polarization is non-zero. Such a drift would be straightforward to incorporate into the classical random walk. The key challenge appears to be the derivation of an analytical expression for Eq. (5) for the case of non-zero bias.

We note that, in modeling the quantum DRF with a semi-classical model, the classical analogue of the quantum state of the frame was a probability distribution over possible classical configurations, i.e., a state of knowledge about the orientation of a classical DRF. This was true even for the initial pure quantum state $|j, j\rangle$. Viewing quantum states as states of knowledge, analogous to classical probability distributions, is the principle tenet of the epistemic view of quantum states [16, 17]. Our results provide further potential for advancing this research program. First, our results demonstrate that, for the specific case of measurements relative to a classical DRF, the degradation of the measurement fidelity is described by a hidden-variable model. In addition, our results highlight the importance of incorporating a form of back-action into the measurement theory within such a hidden-variable model. The existence (and necessity) of 
back-action associated with measurement is also a feature of "toy" models based on the principles of the epistemic view that reproduce quantum-like phenomena [17].

\section{ACKNOWLEDGMENTS}

We wish Peter Knight well on this special occasion and appreciate his support and guidance throughout the years.

The authors gratefully acknowledge J-C Boileau, David Poulin, and Robert Spekkens for helpful discus- sions. SDB acknowledges support from the Australian Research Council (ARC). TR acknowledges support from the Engineering and Physical Sciences Research Council of the United Kingdom, and a University of Sydney Short-Term Visiting Fellowship. BCS acknowledges support from Alberta's Informatics Circle of Research Excellence (iCORE), ARC, and the Canadian Institute for Advanced Research. PST acknowledges support from an iCORE Alberta Ingenuity Fund Fellowship, and from the Network of Centres of Excellence for the Mathematics of Information Technology and Complex systems.
[1] S. D. Bartlett, T. Rudolph and R. W. Spekkens, arXiv:quant-ph/0610030 (2006).

[2] A. Peres and P. F. Scudo, Phys. Rev. Lett. 86, 4160 (2001).

[3] S. J. van Enk and H. J. Kimble, Quant. Inf. Comput. 2, 1 (2002).

[4] J. Gea-Banacloche, Phys. Rev. A 65, 022308 (2002).

[5] T. Tyc and B. C. Sanders, J. Phys. A: Math. Gen. 37, 7341 (2004).

[6] S. D. Bartlett, T. Rudolph, R. W. Spekkens, P. S. Turner, New J. Phys. 8, 58 (2006).

[7] D. Poulin and J. Yard, arXiv:quant-ph/0612126 (2006).

[8] D. Rugar, R. Budakian, H. J. Mamin, and B. W. Chui, Nature (London)430, 329 (2004);

J. A. Sidles, J. L. Garbini, K. J. Bruland, D. Rugar, O. Züger, S. Hoen, and C. S. Yannoni, Rev. Mod. Phys. 67, 249 (1995).

[9] Y. Makhlin, G. Schön, and A. Shnirman, Rev. Mod.
Phys. 73, 357 (2001).

[10] S. D. Bartlett, T. Rudolph and R. W. Spekkens, Phys. Rev. A 70, 032321 (2004).

[11] M. A. Nielsen and I. L. Chuang, Quantum Computation and Quantum Information (Cambridge University Press, Cambridge, 2000).

[12] F. T. Arecchi, E. Courtens, R. Gilmore, and H. Thomas, Phys. Rev. A 6, 2211 (1972).

[13] A. Perelomov, Generalized Coherent States and Their Applications (Springer, Berlin, 1986).

[14] D. J. Rowe, H. de Guise, and B. C. Sanders, J. Math. Phys. 42, 2315 (2001).

[15] P. H. Roberts and H. D. Ursell, Phil. Trans. R. Soc. A 252, 317 (1960).

[16] C. H. Fuchs, arXiv:quant-ph/0205037 (2002); J. Mod. Opt. 50, 987 (2003).

[17] R. W. Spekkens, arXiv:quant-ph/0401052 (2004). 